Tropical Journal of Pharmaceutical Research February 2021; 20 (2): 269-274

ISSN: $1596-5996$ (print); 1596-9827 (electronic)

(C) Pharmacotherapy Group, Faculty of Pharmacy, University of Benin, Benin City, 300001 Nigeria

Available online at http://www.tjpr.org

Original Research Article

http://dx.doi.org/10.4314/tjpr.v20i2.7

\title{
Curcuma longa Linn extract suppresses neuronal apoptosis induction by sevoflurane via activation of the ERK1/2 pathway
}

\author{
Zhou Yu*, Yao Yan, Ying Lou \\ Department of Anesthesiology, The First People's Hospital of Fuyang District, Hangzhou Fuyang District, Hangzhou 311400, \\ China \\ *For correspondence: Email: ZhouYu7655@gmail.com; Tel: 0086-0571-63157231
}

Sent for review: 28 July 2020

Revised accepted: 20 January 2021

\begin{abstract}
Purpose: To investigate Curcuma longa Linn against neuronal damage induced by exposure to sevoflurane during surgical procedures.

Methods: A sealed box made of transparent glass was used for anaesthetic exposure of neurons. The neurons were exposed to Curcuma longa Linn at doses of 1.5, 3, 6 and $12 \mu \mathrm{M}$ prior to viability assessment using MTT assay. The effect of Curcuma longa Linn treatment on protein expression was determined using western blotting.

Results: Sevoflurane exposure led to significant and time-dependent reductions in neuronal proliferation, when compared to unexposed cells $(p<0.05)$. Curcuma longa Linn at doses of 1.5, 3, 6 and $12 \mu \mathrm{M}$ significantly decreased sevoflurane-mediated neuronal apoptosis. It reduced cleaved caspase-3 and Bax levels in neurons. However, the Curcuma longa Linn-mediated inhibition of sevoflurane-induced neuronal apoptosis was significantly suppressed by VPC23019 $(p<0.05)$. The $p$ $E R K 1 / 2$ level was dose-dependently up-regulated in neurons exposed to sevoflurane on treatment with Curcuma longa Linn. Moreover, VPC23019 reversed the upregulatory effect of Curcuma longa Linn on $p$-ERK1/2 expression in sevoflurane-exposed neurons $(p<0.05)$.

Conclusion: Curcuma longa Linn reversed sevoflurane-induced neuronal apoptosis by elevating $p$ ERK1/2 expression. Therefore, Curcuma longa Linn exerts inhibitory effect on anaesthesia-induced apoptosis in neurons, and may be useful for the treatment of this condition.
\end{abstract}

Keywords: Anesthesia, Neurotoxicity, Sphingomyelin, Apoptosis, Curcuma longa

\begin{abstract}
This is an Open Access article that uses a fund-ing model which does not charge readers or their institutions for access and distributed under the terms of the Creative Commons Attribution License (http://creativecommons.org/licenses/by/4.0) and the Budapest Open Access Initiative (http://www.budapestopenaccessinitiative.org/read), which permit unrestricted use, distribution, and reproduction in any medium, provided the original work is properly credited.

Tropical Journal of Pharmaceutical Research is indexed by Science Citation Index (SciSearch), Scopus, International Pharmaceutical Abstract, Chemical Abstracts, Embase, Index Copernicus, EBSCO, African Index Medicus, JournalSeek, Journal Citation Reports/Science Edition, Directory of Open Access Journals (DOAJ), African Journal Online, Bioline International, Open-J-Gate and Pharmacy Abstracts
\end{abstract}

\section{INTRODUCTION}

Anaesthesia is most frequently used in surgical procedures for the convenience of patents, and also in response to patients' demand $[1,2]$. However, increased application of anaesthesia has led to many complications in patients, including cognitive dysfunction following surgery [3]. Due to these complications, the effect of anaesthetic compounds on the nervous system, especially their effects on memory and cognition potential in children and infants, have been investigated [4]. The most frequently used anaesthetic compound for surgeries is 
sevoflurane, due to its stable hemodynamics, quick patient recovery, and negligible deleterious impact on the kidneys and liver [5]. Studies have found that sevoflurane inhibits proliferative potential of cortical progenitor cells and promotes neuronal death in cortex of CNS [6]. Thus, sevoflurane causes memory impairment and loss of cognition in new-born animal models [9]. It has been demonstrated that sevoflurane induces toxicity and neuronal apoptosis which subsequently result in cognitive impairment $[12,13]$. Thus, there is need for prevention of sevoflurane-induced neurotoxicity so as to inhibit memory loss. Sphingomyelin is an important part of oligodendrocytes and myelin covering in the CNS [7]. The conversion of sphingomyelin to ceramide by phospholipase $\mathrm{C}$ is followed by its transformation to sphingosine by the enzyme ceramidase [8]. Phosphorylation of sphingosine leads to its conversion to sphingosine-1phosphate $(\mathrm{S} 1 \mathrm{P})$ in a reaction catalyzed by sphingosine kinase (SphK) [9]. The survival and proliferation of neurons are associated with the activation of S1P signal pathway [10]. Neuraminic acid treatment has been demonstrated to upregulate the S1P pathway, thereby targeting apoptosis in PC12 cells [10]. Impaired transmission of the sphingosine kinase 1/S1P signal pathway causes disruption in neuronal cell survival and growth, resulting in defective neural progenitor cells [11].

Curcuma longa Linn (CL) grows in tropical and subtropical regions world-wide [12]. The plant has traditional medicinal importance with respect to healing of wounds, and treatment of infectious fever and liver diseases [12]. Moreover, it has anti-inflammatory potential [13], hepatoprotective property [13] and anti-arthritic uses. Present study investigated Curcuma longa Linn against neuronal damage induced by exposure to sevoflurane during surgical procedures.

\section{EXPERIMENTAL}

\section{Preparation of hippocampal neurons}

Sprague-Dawley rats (20 day old; body weight = $48 \mathrm{~g})$ were provided by the Animal Centre of Beijing (Beijing, China). Temperature was maintained at $22 \pm 2{ }^{\circ} \mathrm{C}$, relative humidity $40 \%$ and day/night durations were controlled to $12-\mathrm{h}$ 112-h. Laboratory feed and water was made freely available to the rats in animal center. The procedures used were carried out in aordane with international guidelines [14]. Following sacrifice through decapitation, the skin around each rat skull was carefully removed, followed by separation of the cerebral hemispheres. The excised tissues were put in petri dishes, and the cortex was isolated to expose and remove the hippocampus. Following washing in Hank's D solution, the tissues were subjected to centrifugation at $200 \times \mathrm{g}$ for $10 \mathrm{~min}$ at $4{ }^{\circ} \mathrm{C}$. Decantation of supernatant was followed by digestion with $10 \mu \mathrm{L}$ of protease for $15-20 \mathrm{~min}$. Thereafter, DMEM and $10 \%$ FBS were added, and the cells were subjected to filtration through 200-mesh size copper filter. Centrifugation and DMEM addition were followed by culture of the single cell suspension in culture flasks at $37^{\circ} \mathrm{C}$.

\section{Treatment of neurons}

Neurons seeded at $2 \times 10^{6}$ cells/ well distribution in 96- well plates were cultured for $24 \mathrm{~h}$. Thereafter, the neurons were incubated overnight for $24 \mathrm{~h}$ with Curcuma longa Linn at doses of 1.5, 3, 6 and $12 \mu \mathrm{M}$; VPC23019 (0.5 $\mu \mathrm{mol} / \mathrm{L})$ and $\mathrm{U} 0126(5 \mu \mathrm{mol} / \mathrm{L})$. Study approval by obtained from the Ethics Committee for Animal Use, The First People's Hospital of Fuyang District (Fuyang, China; approval no. = UU/002/18). All experiments were conducted according to the guidelines issued by the National Institute of Health China.

\section{Exposure to anaesthesia}

A sealed box made of transparent glass was used for exposure of the neurons to anesthesia. The box contained $100 \mathrm{~g}$ of soda lime at the bottom, and apertures on the lateral sides were connected to anaesthesia machine (Dräger Fabius ${ }^{\circledR}$ GS Premium, Germany) using threaded pipe. The primary hippocampal neurons were distributed in Matrigel basement coated 24- well plates at a density of $2 \times 10^{5}$ cells/well in neurobasal medium. The neurons were exposed to Curcuma longa Linn at doses of $1.5,3,6$ and $12 \mu \mathrm{M}$, after which they were subjected to tetrazolium salt (3-(4,5dimethylthiazol-2-yl)-2,5-diphenyltetrazolium bromide (MTT) assay.

\section{Cell viability assay}

The neuronal cells were seeded in 96-well plates at a density of $2 \times 10^{5}$ cells/well, and were cultured in a $5 \% \mathrm{CO}_{2}$ incubator at $37^{\circ} \mathrm{C}$. The neurons were exposed to Curcuma longa Linn at doses of $1.5,3,6$ and $12 \mu \mathrm{M}$; and $0.5 \mu \mathrm{mol} / \mathrm{L}$ VPC23019 for $24 \mathrm{~h}$. Thereafter, $20 \mu \mathrm{L}$ of MTT solution $(5 \mathrm{mg} / \mathrm{mL})$ was added to each of the wells, and incubation was continued for additional $4 \mathrm{~h}$. The formazan crystals formed were dissolved by addition of $150 \mu \mathrm{L}$ of DMSO to each well. The absorbance of the solution in each well was read at $488 \mathrm{~nm}$ in an enzymelinked immunodetector. 


\section{Western blot analysis}

The medium was discarded from the plates and the cells were washed, agitated and subsequently lysed in radioimmunoprecipitation buffer for $45 \mathrm{~min}$. The lysate was transferred with a pipette into a centrifuge tube and centrifuged at $1,200 \times \mathrm{g}$ for $10 \mathrm{~min}$ at $4{ }^{\circ} \mathrm{C}$. The protein content of each lysate was estimated using BCA assay. Then, $50-\mu \mathrm{g}$ samples were resolved on SDS-PAGE, followed by transfer onto PVDF membranes which were blocked using $5 \%$ non-fat milk solution. Thereafter, the membranes were incubated overnight with the primary antibodies antiBax, anti-caspase-3, anti$\mathrm{Bcl} 2$, anti-p-ERK, anti-ERK and anti- $\beta$-actin at 4 ${ }^{\circ} \mathrm{C}$. Then, the membranes were washed and incubated for $2 \mathrm{~h}$ with goat anti-rabbit IgG secondary antibody at $37{ }^{\circ} \mathrm{C}$. The proteins were visualized using ECL system connected to Odyssey far-infrared fluorescence system (LICOR Biosciences, USA).

\section{Statistical analysis}

Data are expressed as mean \pm SD of triplicate measurements. Statistical analysis of the data was made using SPSS 19.0 statistical software. One-way analysis of variance (ANOVA) and post-hoc test were used for comparison of data. Differences were considered statistically significant at $p<0.05$.

\section{RESULTS}

\section{Sevoflurane inhibits neuron viability}

Exposure of neurons to sevoflurane for 2, 4, 8, 16 and $32 \mathrm{~h}$ was followed by evaluation of cell proliferation (Figure 1). There was a significant and time-dependent reduction in the proliferation of neurons exposed to sevoflurane, relative to unexposed neurons $(p<0.05)$. Neuronal proliferation was reduced to $91,83,70,56$ and $49 \%$, by sevoflurane at $2,4,8,16$ and $32 \mathrm{~h}$, respectively.

\section{Curcuma longa Linn prevented apoptosis by sevoflurane in neurons}

Curcuma longa Linn treatment prevented sevoflurane-mediated activation of apoptosis in neurons in a dose-dependent manner (Figure 2). Significant decreases in sevoflurane-mediated apoptosis by Curcuma longa Linn treatment were observed at doses of 1.5, 3, 6 and $12 \mu \mathrm{M}$. At a dose of $12 \mu \mathrm{M}$, Curcuma longa Linn treatment suppressed sevoflurane-mediated neuronal apoptosis to the lowest level.

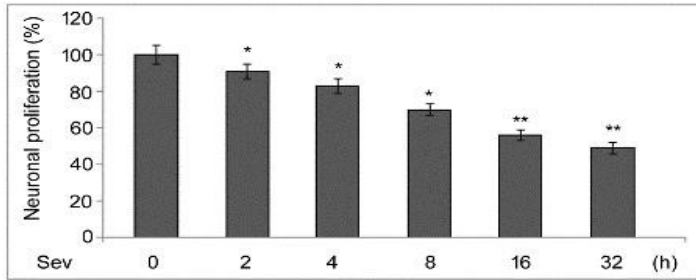

Figure 1: Effect of sevoflurane on proliferation of neurons. Neurons exposed to $3 \%$ sevoflurane for 2 , $4,8,16$ and $32 \mathrm{~h}$, and unexposed neurons were examined for proliferative changes using MTT assay. ${ }^{\star} P<0.05,{ }^{* *} p<0.02$ vs. sevoflurane group

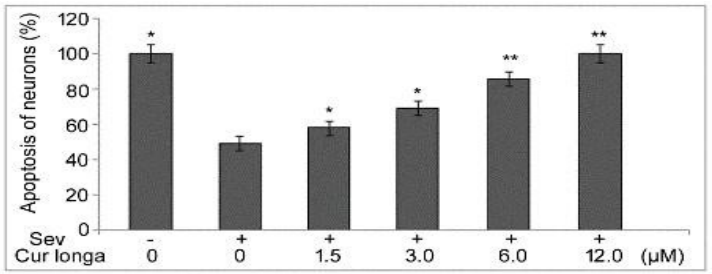

Figure 2: Effect of Curcuma longa Linn on sevoflurane-activated apoptosis of neurons. Treatment of neurons with Curcuma longa Linn at doses 1.5, 3, 6 and $12 \mu \mathrm{M}$ after sevoflurane exposure was followed apoptosis analysis. ${ }^{*} P<0.05 ;{ }^{* *} p<0.02$, vs. sevoflurane group

Curcuma longa Linn inhibited sevofluranemediated expressions of apoptotic proteins in neurons

Treatment with Curcuma longa Linn reduced levels of cleaved caspase- $3 \mathrm{C}$ and Bax in neurons exposed to sevoflurane (Figure 3 ). The levels of $\mathrm{Bcl}-2$ in neurons exposed to sevoflurane were elevated on treatment with Curcuma longa Linn.

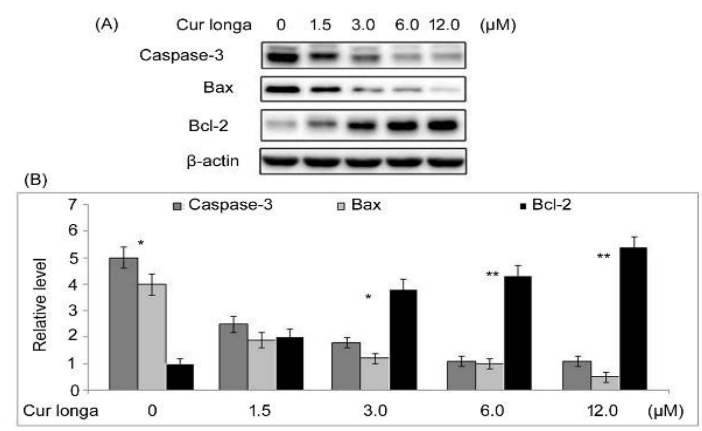

Figure 3: Curcuma longa Linn suppresses expressions of apoptosis-related proteins. (A) Treatment of neurons with Curcuma longa Linn at doses of $1.5,3,6$ and $12 \mu \mathrm{M}$ after sevoflurane exposure was followed by Western blotting. (B) Protein band quantification levels. ${ }^{*} P<0.05$; ${ }^{* *} p<$ 0.02 , vs. sevoflurane group 


\section{VPC23019 reversed Curcuma longa- mediated anti-apoptotic effect in neurons exposed to sevoflurane}

Curcuma longa Linn-mediated antiapoptotic effect in neurons exposed to sevoflurane was markedly suppressed by VPC23019 (Figure 4). Moreover, VPC23019 upregulated cleaved caspase-3 cleavage and Bax level in Curcuma longa Linn-treated and sevoflurane-exposed neurons. The $\mathrm{Bcl} 2$ level in Curcuma longa Linntreated and sevoflurane-exposed neurons was effectively reduced by VPC23019.

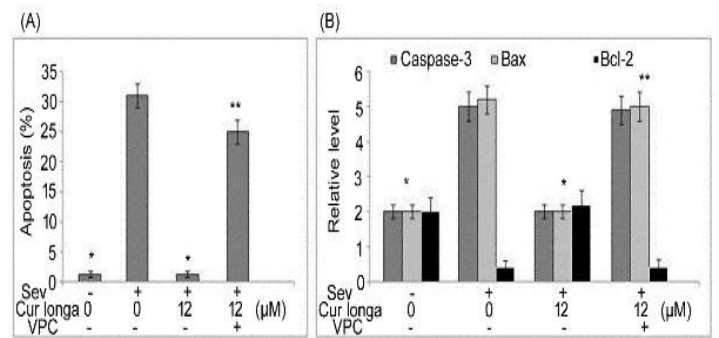

Figure 4: Effect of VPC23019 on Curcuma longa Linn-mediated inhibition of apoptosis. (A) Apoptosis in untreated, sevoflurane-exposed, Curcuma longa Linntreated + sevoflurane exposed, and Curcuma longa Linn-treated + sevoflurane-exposed + VPC23019treated groups of neurons, as measured using MTT assay. (B) Protein levels. ${ }^{*} P<0.05,{ }^{* *} p<0.02$, vs. sevoflurane group

\section{U0126 reversed Curcuma longa Linn- mediated inhibition of apoptotic changes due to sevoflurane}

Inhibition of apoptosis by Curcuma longa Linn in neurons exposed to sevoflurane was effectively suppressed by U0126 (Figure 5). Compared to Curcuma longa Linn-treated neurons, apoptosis was significantly increased by $\mathrm{U} 0126$ in Curcuma longa Linn-treated and sevofluraneexposed neurons. Moreover, U0126 elevated cleaved caspase- 3 and Bax levels in Curcuma longa Linn-treated and sevoflurane-exposed neurons, relative to Curcuma longa Linn-treated neurons. In addition, U0126 suppressed Bcl2 expression in neurons treated with Curcuma longa Linn., relative to neurons treated with Curcuma longa Linn. and sevoflurane.

\section{Curcuma longa Linn upregulated p-ERK1/2 expression}

In neurons exposed to sevoflurane, there was a marked reduction in $\mathrm{p}$-ERK $1 / 2$ protein, when compared to control cells (Figure 6). In contrast, p- ERK1/2 protein was markedly and dosedependently upregulated by Curcuma longa Linn in neurons exposed to sevoflurane. However,
VPC23019 reversed the up-regulatory role of Curcuma longa Linn on p-ERK1/2 expression in neurons exposed to sevoflurane.

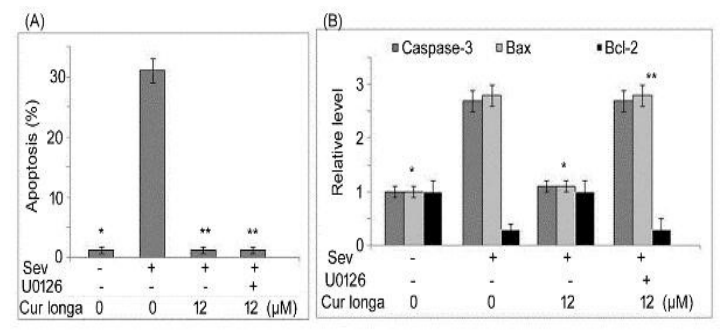

Figure 5: Effect of U0126 on Curcuma longamediated inhibition of apoptosis. (A) Apoptosis in untreated, sevoflurane-exposed, Curcuma longa Linntreated + sevoflurane-exposed, and Curcuma longa Linn-treated + sevoflurane-exposed + U0126-treated groups of neurons, as measured using MTT assay. (B) Protein levels of apoptotic factors in the various groups of neurons. ${ }^{*} P<0.05$, ${ }^{*} p<0.02$, vs. sevoflurane group

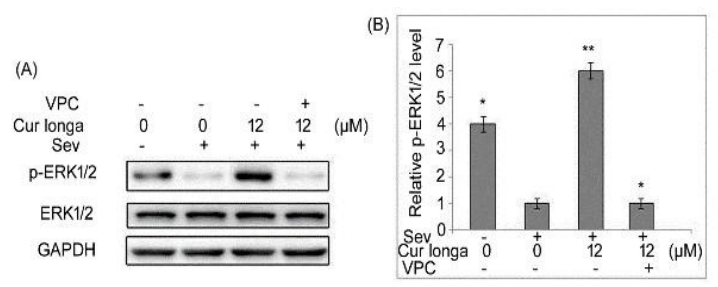

Figure 6: Effects of Curcuma longa Linn and VPC23019 on expression of p-ERK1/2. (A) Expressions of $\mathrm{p}-\mathrm{ERK} 1 / 2$ in untreated, sevofluraneexposed, Curcuma longa Linn-treated + sevofluraneexposed, and Curcuma longa Linn-treated + sevoflurane-exposed + VPC23019-treated groups of neurons, as measured using Western blotting. (B) Quantified protein levels of $p$-ERK $1 / 2$ in the various groups; ${ }^{*} p<0.05 ;{ }^{* *} p<0.02$, vs. sevoflurane group

\section{DISCUSSION}

Sevoflurane, an anaesthetic compound used during surgeries, has been reported to induce apoptosis in neurons and subsequent impairment of memory and cognitive potential [15]. Sevoflurane-induced neuronal damage has been reported in Alzheimer's disease patients as well as in healthy people [16]. Many pathways associated with signal transduction such as NF$\mathrm{K}-\mathrm{B}$ and S1P have been found to be involved in sevoflurane-induced neurological damage [17]. In agreement with findings in previous studies, the present study has revealed that exposure of neurons to sevoflurane resulted in timedependent suppression of proliferation. Neuronal proliferation was reduced to $91,83,70,56$ and $49 \%$ by sevoflurane at $2,4,8,16$ and $32 \mathrm{~h}$, respectively. Activation of sevoflurane-induced neuronal apoptosis was inhibited in a dose- 
dependent manner on treatment with Curcuma longa Linn. It has been demonstrated that the sphingosine-1-phosphate (S1P) pathway enhances the growth and proliferation of neurons [18]. The activation of SIPI by chemotherapeutics has been reported to mitigate induction of neuronal apoptosis by anaesthetic compounds [19]. Since S1P1 activation is involved in signal transductions required for survival of neurons, a study determined effect of VPC23019 (S1P1 antagonist) on Curcuma longa Linn-mediated suppression of apoptosis in neurons exposed to sevoflurane [10]. In the present study, Curcuma longa Linn-mediated inhibition of apoptosis in neurons exposed to sevoflurane was prevented significantly by VPC23019. Moreover, VPC23019 upregulated the expressions levels of cleaved caspase-3 and Bax in Curcuma longa Linntreated and sevoflurane-exposed neurons. The Bcl2 level in Curcuma longa Linn-treated and sevoflurane-exposed neurons was effectively reduced by VPC23019. These data indicate that Curcuma longa Linn suppressed apoptosis in sevoflurane-exposed neurons by activation of the S1P1 pathway.

Anaesthetic compounds generally target ERK which is the major pathway involved in neuronal apoptosis [20, 21]. Neuronal viability is maintained by $\mathrm{p}$ - ERK $1 / 2$ during exposure to anaesthetic compounds [22]. In the present study, exposure to sevoflurane led to reduction in $\mathrm{p}$ - ERK1/2 protein in neurons, relative to unexposed neurons. The $\mathrm{p}$ - ERK1/2 level was promoted markedly in neurons exposed to sevoflurane by Curcuma longa Linn treatment. However, VPC23019 reversed the Curcuma longa Linn mediated enhancement in p- ERK1/2 expression in neurons exposed to sevoflurane.

\section{CONCLUSION}

Curcuma longa Linn extract reverses sevoflurane-induced apoptosis in neurons by upregulating the ERK1/2 phosphorylation. The S1P1 pathway in sevoflurane-exposed neurons is also enhanced by Curcuma longa treatment. These results suggest that the extract exerts protective effect against anaesthesia-induced apoptosis in neurons. Thus, it may be beneficial in the treatment of neuronal apoptosis induced by anesthetics.

\section{DECLARATIONS}

\section{Conflict of interest}

No conflict of interest is associated with this work.

\section{Contribution of authors}

We declare that this work was done by the authors named in this article and all liabilities pertaining to claims relating to the content of this article will be borne by the authors. Yao Yan and Ying Lou performed the experimental work, carried out the literature survey and analysed and compiled the data. Zhou $\mathrm{Yu}$ designed the study and wrote the manuscript. All authors read the paper thoroughly and approved it for publication.

\section{Open Access}

This is an Open Access article that uses a funding model which does not charge readers or their institutions for access and distributed under the terms of the Creative Commons Attribution License (http://creativecommons.org/licenses/by/ 4.0) and the Budapest Open Access Initiative (http://www.budapestopenaccessinitiative.org/rea d), which permit unrestricted use, distribution, and reproduction in any medium, provided the original work is properly credited.

\section{REFERENCES}

1. Wan Hassan WMN, Tan HS, Mohamed Zaini RH. Comparison of the effects of dexmedetomidine on the induction of anaesthesia using marsh and schnider pharmacokinetic models of propofol Target Controlled infusion. Malays J Med Sci 2018; 25: 2431.

2. Afolayan JM, Areo PO, Adegun PT, Ogundipe KO, Filani $A B$. Comparison of ease of induction of spinal anaesthesia in sitting with legs parallel on the table versus traditional sitting position. Pan Afr Med J 2017; 28: 223.

3. Anderson BJ. Drug error in paediatric anaesthesia: Current status and where to go now. Curr Opin Anaesthesiol 2018; 31: 333341.

4. Gautam B, Niroula S, Sharma M, Lama SM. Effects of intrathecal dexmedetomidine as an adjuvant to hyperbaric bupivacaine for spinal anaesthesia in adults undergoing elective infra umbilical surgery. JNMA J Nepal Med Assoc 2017; 56: 379387.

5. Juodzente D, Macas A, Karveliene B, Petkevicius S, Riskeviciene V. Comparison of the cardiovascular and respiratory effects and sevoflurane requirement in dogs premedicated with two doses of medetomidine and butorphanol undergoing surgical sterilization. Pol J Vet Sci 2018; 21: 101110.

6. Zhang LM, Zhang DX, Zhao XC, Sun W. Erythropoietin rescues primary rat cortical neurons by altering the nrf2:Bach1 ratio: Roles of extracellular Signal Regulated kinase 1/2. Neurochem Res: 2017; Jan 12 (Epub ahead of print) 
7. Jang YE, Jeong SA, Kim SY, Song IK, Lee JH, Kim JT, Kim HS. The efficacy of intraoperative EEG to predict the occurrence of emergence agitation in the postanesthetic room after sevoflurane anesthesia in children. J Perianesth Nurs 2018; 33: 4552.

8. Lu G, Xu H, Zhao W, Zhang J, Rao D, Xu S. Upregulation of long noncoding RNA Gadd45a is associated with sevoflurane induced neurotoxicity in rat neural stem cells. Neuroreport 2018; 29: 605614.

9. Szepanowski F, Derksen A, Steiner I, Meyer Zu Hörste $G$, Daldrup T, Hartung HP, Kieseier BC. Fingolimod promotes peripheral nerve regeneration via modulation of lysophospholipid signaling. J Neuroinflammation 2016; 13: 143

10. Cho MC, Park K, Chai JS, Lee SH, Kim SW, Paick JS. Involvement of sphingosine 1 phosphate/RhoA/Rho kinase signaling pathway in corporal fibrosis following cavernous nerve injury in male rats. J Sex Med 2011; 8: 712721.

11. Costello RW, Maloney M, Atiyeh M, Gleich G, Walsh MT. Mechanism of sphingosine 1 phosphate and lysophosphatidic acid induced up regulation of adhesion molecules and eosinophil chemoattractant in nerve cells. Int J Mol Sci 2011; 12: 32373249.

12. Araújo CC, Leon LL. Biological activities of Curcuma Ionga L. Mem Inst Oswaldo Cruz. 2001; 96: 723-728.

13. Park EJ, Jeon $C H, K o ~ G, K i m ~ J$, Sohn DH. Protective effect of curcumin in rat liver injury induced by carbon tetrachloride. J Pharm Pharmacol 2000; 52: 437-40.

14. Guide for the Care and Use of Laboratory Animals: Eighth Edition Committee for the Update of the Guide for the Care and Use of Laboratory Animals; National Research Council. 2010; ISBN: 0-309-15401-4

15. Wen $T$, Wang L, Sun $X J$, Zhao $X$, Zhang GW, Li Ling J. Sevoflurane preconditioning promotes activation of resident CSCs by transplanted BMSCs via miR 210 in a rat model for myocardial infarction. Oncotarget 2017; 8: 114637114647.

16. He $H$, Liu W, Zhou Y, Liu Y, Weng P, Li Y, Fu H. Sevoflurane post conditioning attenuates traumatic brain injury induced neuronal apoptosis by promoting autophagy via the PI3K/AKT signaling pathway. Drug Des Devel Ther 2018; 12: 629638.

17. Zhang $Y H$, Vasko MR, Nicol GD. Intracellular sphingosine 1 phosphate mediates the increased excitability produced by nerve growth factor in rat sensory neurons. J Physiol 2006; 575: 101113.

18. Nicol GD. Nerve growth factor, sphingomyelins, and sensitization in sensory neurons. Sheng $\mathrm{Li}$ Xue Bao 2008; 60: 603604.

19. Bayes M, Rabasseda X, Prous JR. Gateways to clinical trials. Methods Find Exp Clin Pharmacol 2002; 24: 217 248.

20. Wang S, Zhou Y. Baicalein inhibits neuroapoptosis via pathways in sevoflurane induced rats. Transl Neurosci 2018; 9: 8898.

21. Osinde M, Mullershausen F, Dev KK. Phosphorylated FTY720 stimulates ERK phosphorylation in astrocytes via S1P receptors. Neuropharmacology 2007; 52: 1210 1218.

22. Liu J, Zhang $X$, Zhang W, Gu G, Wang P. Effects of sevoflurane on young male adult C57BL/6 mice spatial cognition. PLoS One 2015; 10: e13422. 